\title{
A Toolkit to Quantify Target Compounds in Thin-Layer- Chromatography Experiments
}

\author{
Niamh Mac Fhionnlaoich, ${ }^{\dagger}{ }^{\circledR}$ Stuart Ibsen, ${ }^{\dagger}$ Luis A. Serrano, ${ }^{\circledR}$ Alaric Taylor, ${ }^{\circledR}$ Runzhang Qi, ${ }^{\circledR}$ \\ and Stefan Guldin*(1)
}

Department of Chemical Engineering, University College London, Torrington Place, London WC1E 7JE, U.K.

\section{Supporting Information}

\begin{abstract}
Thin-layer chromatography (TLC) is one of the basic analytical procedures in chemistry and allows the demonstration of various chemical principles in an educational setting. An often-overlooked aspect of TLC is the capability to quantify isolated target compounds in an unknown sample. Here, we present a suitable route to implement quantitative analysis in a lesson plan. We provide both a stand-alone software and an online webapp that allow students to obtain quantitative information from a developed TLC plate and present two suitable experiments, namely, the absorbance-based quantification of the colorant Sudan IV and the fluorescence-based quantification of rhodamine 6G, a fluorophore widely used in biotechnology. Students conduct TLC experiments following established protocols, take pictures of their TLC plates with mobile phones, and subsequently quantify the different compounds in the separate bands they observe.

KEYWORDS: General Public, High School/Introductory Chemistry, First-Year Undergraduate/General,

Second-Year Undergraduate, Upper-Division Undergraduate, Graduate Education/Research, Continuing Education,

Analytical Chemistry, Demonstrations, Environmental Chemistry, Laboratory Instruction, Public Understanding/Outreach,

Computer-Based Learning, Hands-On Learning/Manipulatives, Inquiry-Based/Discovery Learning, Problem Solving/Decision Making,

Laboratory Computing/Interfacing, Laboratory Equipment/Apparatus, Microscale Lab, Quantitative Analysis,

Student-Centered Learning, Thin Layer Chromatography
\end{abstract}

\section{INTRODUCTION}

Thin-layer chromatography (TLC) is a well-known chemicalanalysis procedure that is inexpensive and simple to perform. The technique involves spotting a sample solution onto a TLC plate, the stationary phase, and placing the plate into a vessel containing the mobile phase. Capillary forces draw the mobile phase through the plate, which drives the chromatographic separation of the individual nonvolatile components of the sample mixture. Once the TLC plate is dried, individual bands may be observed via absorbance or fluorescence. ${ }^{1,2}$

TLC has been thoroughly developed for use in an educational setting. ${ }^{3-8}$ Dickson et al. proposed a series of simple laboratory experiments designed to teach students the fundamentals of TLC, such as the roles of the retardation factor, hydrogen bonding, and polarity. ${ }^{3}$ Six suitable separations of mixtures of organic compounds and inorganic ions were developed by Brinkman and De Vries. ${ }^{4}$ Olensen and Hopson presented an experiment whereby students used principles of TLC to identify the composition of an unknown black ink. ${ }^{5} \mathrm{Ma}$ and Yeung designed a route for the separation of components from beverages. ${ }^{6}$ Building on this practical example, Torres y Torres et al. proposed extraction and analysis of caffeine from beverages using TLC. ${ }^{7}$ Recently, Sjurnes et al. outlined an experiment to teach normal and reverse-phase TLC using an example of green-leaf extracts. ${ }^{8}$

The quantification of target compounds is typically not included in these lesson plans because of a lack of inexpensive and easy-to-use tools that are compatible with TLC and designed for an educational setting. To this end, Valverde et al. introduced a route to quantify the photosynthetic pigments in green beans by using a flatbed scanner. ${ }^{9}$ Although this work provides an interesting approach to densitometric quantification, the integration of flatbed scanners into suitable workstations and safe experimental conduct remains challenging. Furthermore, the method lacks compatibility with fluorimetry, and staining of target compounds is only available for some colorless compounds. ${ }^{10}$ An alternative route to obtain images suitable for quantitative analysis is digital photography. Hess introduced an approach compatible with classroom settings and provided an appropriate algorithm. ${ }^{11}$ Further developments were aimed toward research applications. Fichou et al. recently presented a web-based application for quantitative TLC analysis (rTLC). ${ }^{12}$ Meanwhile, ImageJ provides a tool for electrophoretic gels, which can easily be applied to TLCs. ${ }^{13,14}$ Although these works and the accompanied software represent important contributions to quantitative read-out and promote their accessibility, limitations remain, especially for educational settings. In all three of the above methods, the quantification routine reduces the two-dimensional (2D) densitogram to a one-dimensional (1D) array for calculation purposes. This loss of dimensional information limits the achievable accuracy and promotes user errors. Furthermore, no accommodation for uneven illumination is made by the software, and any thresholding is applied on a local basis, which will negatively affect the consistency of the analysis across the TLC plate. Both, the rTLC and ImageJ

Received: February 26, 2018

Revised: September 6, 2018 
a

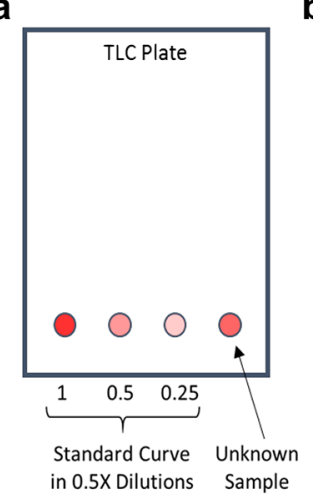

b

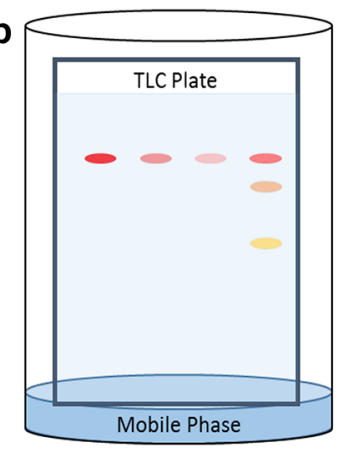

c

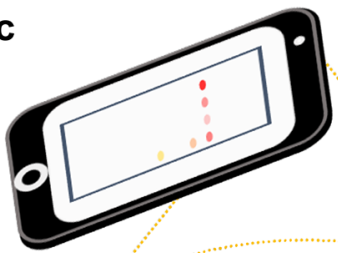

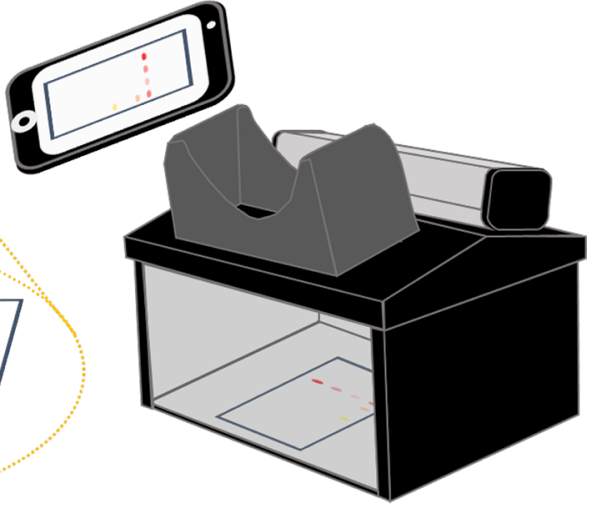

Figure 1. TLC-plate preparation. (a) Serial dilution of the target compound spotted onto the plate along with the unknown sample. (b) Development of the TLC plate in a chamber containing the mobile phase in order to elute the sample. (c) Direct imaging of the absorbing compounds with a mobile phone or other digital camera once the plate has dried. For nonabsorbing but fluorescent samples, a black box containing the respective excitation light may be used.

software require a consistent lane width and the rTLC further demands regular lane spacing; this is difficult to achieve in an educational setting where users are less experienced with TLCsample preparation.

Here, we present an intuitive and user-friendly analytic platform that enables students to reliably quantify the compounds they are studying. Additionally, we supply specifically designed and freely available software for Windows and MacOS operating systems and an online webapp for all mainstream browsers. ${ }^{15}$ These programs are developed to work with images obtained by nonspecialized equipment, which can be as simple as a mobile phone. Uniquely, the tools identify overexposed pixels and allow for correction of uneven illumination. The image-analysis routines enable students to build their own calibration curves using known dilutions of a stock solution and to quantify the target compound in a sample of unknown concentration. By placing the calibration curve and the "unknown sample" on the same TLC plate, more accurate results can be obtained. The tools require the user to select the bands of interest and then automatically determine their physical dimensions and intensities in 2D. This removes user error and bias from the process of quantification. Furthermore, no assumptions are necessary in terms of lane width or spacing. We present two example experiments and provide step-by-step guides for successful implementation. The first experiment relates to the colorant Sudan IV for absorbance-based quantification with relevance in analytical environmental chemistry. In a second experiment, rhodamine $6 \mathrm{G}$, a fluorophore widely used in biotechnology, is quantified by fluorimetric means. Although these two experiments demonstrate the capabilities of the toolkit, the analytic approach is generic and can be applied to a wide variety of other TLC experiments.

\section{EXPERIMENTAL SECTION}

\section{Materials}

Acetic acid (99.8\%), methanol ( $\mathrm{MeOH}, \mathrm{HPLC}$ grade), rhodamine 6G (99\%, CAS number 989-38-8), and Sudan IV (CAS number 85-83-6) were purchased from Sigma-Aldrich and used without further purification. Nonfluorescent, aluminum-backed, normal-phase TLC plates (TLC silica gel 60) were obtained from Merck.

\section{TLC-Plate Preparation}

The TLC separation may be performed using any of the wellestablished TLC lesson plans already available in the literature. $^{3-8}$ An appropriate schematic protocol is shown in Figure 1.

Herein, TLC plates were carefully cut in squares of $5 \times 5 \mathrm{~cm}$ (width $\times$ height). Other dimensions may be used depending on instructor preference and available resources (e.g., $5 \times 10$ $\mathrm{cm})$. A horizontal line was drawn with a pencil $1.0 \mathrm{~cm}$ above the bottom of the plate to define the spotting level and $0.5 \mathrm{~cm}$ below the top of the TLC plate to indicate an upper limit for elution. At least three different concentrations of the target compound were spotted on the plate in order to produce a calibration curve. An equal volume of the unknown-sample solution was then applied to the right of the calibration points. After elution in a dedicated chamber, the TLC plates were dried on a flat surface at room temperature for at least $5 \mathrm{~min}$ and subsequently imaged.

In Experiment 1, suitability for absorbance-based quantification was demonstrated with the compound Sudan IV. Concentrations of 50,25, and $10 \mu \mathrm{M}$ in $\mathrm{MeOH}$ were prepared for the standard curve, and a concentration of $35 \mu \mathrm{M}$ in $\mathrm{MeOH}$ for the unknown sample. Errors associated with these values are discussed in the Supporting Information (SI). A volume of $5 \mu \mathrm{L}$ was used for each spot using a pipette. Note that consistent volume dosing is of high importance for the quantification. An equally suitable method involves the use of capillary tubes, which are weighed before and after filling to determine the quantity used. ${ }^{9,11}$ The experiments were performed on a standard TLC plate with a mobile phase of 1 vol \% acetic acid in $\mathrm{MeOH}$. The absorbance of Sudan IV was found to be linear up to $50 \mu \mathrm{M}$ (see the SI, Figure S7).

In Experiment 2, the compatibility of the platform for fluorescence-based quantification was validated with the compound rhodamine $6 \mathrm{G}$. Concentrations of 10,5 , and 2 $\mu \mathrm{M}$ in $\mathrm{MeOH}$ were prepared for the standard curve, and a concentration of $6 \mu \mathrm{M}$ for the unknown sample. Volumes of 5 $\mu \mathrm{L}$ were applied for each spot. Standard TLC plates were used with a mobile phase of 1 vol \% acetic acid in $\mathrm{MeOH}$. The fluorescent signal of rhodamine $6 \mathrm{G}$ was found to be linear up to $10 \mu \mathrm{M}$ (see SI, Figure S8).

We refer to the SI for a sample student handout on TLC experimentation that contains learning objectives, theory, 

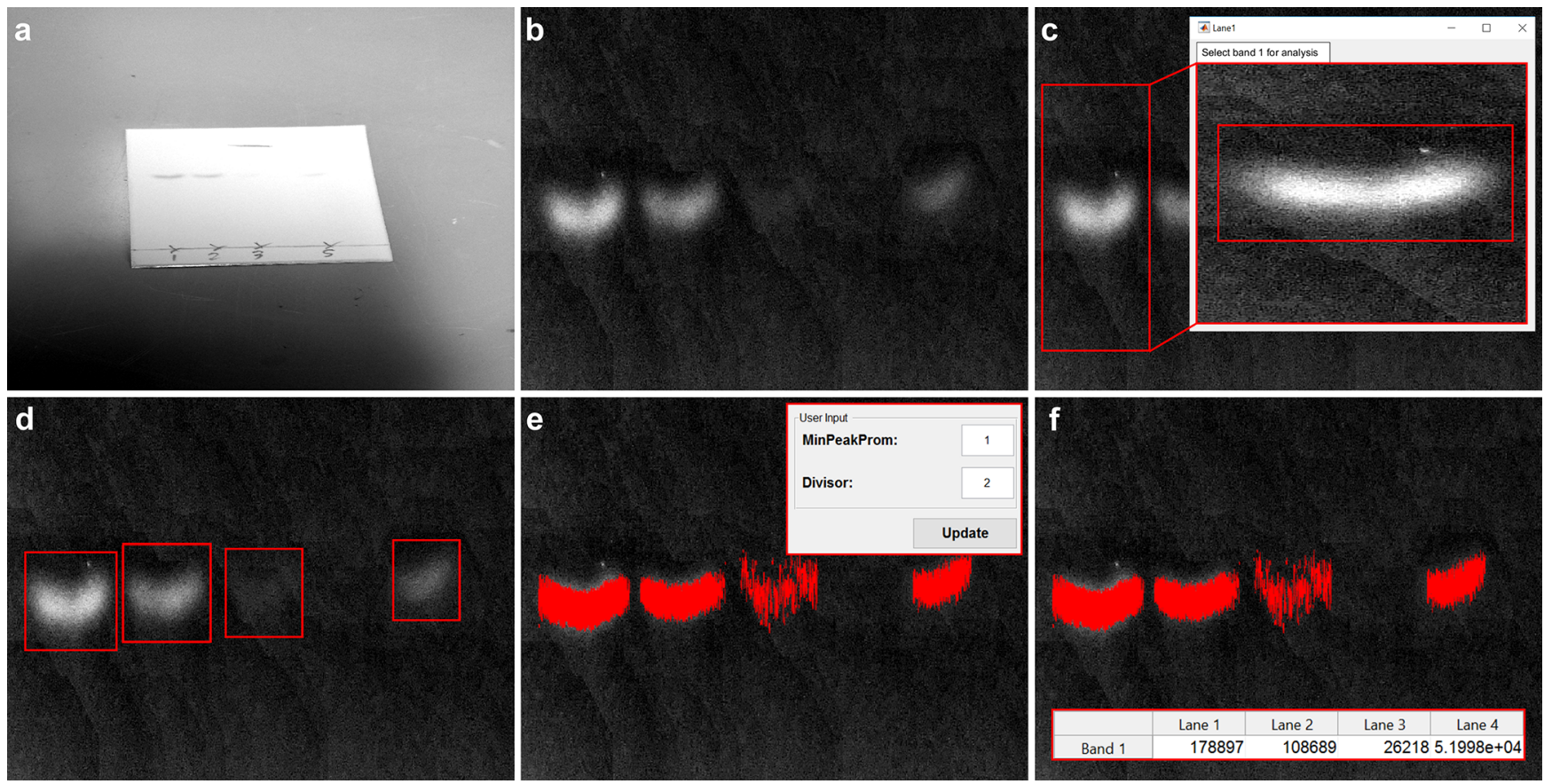

Figure 2. Overview of software implementation. (a) The image of the TLC plate is uploaded into the software. (b) The image is cropped, colors inverted and background correction applied. (c) The lane and band numbers are specified and lanes and bands selected. (d) The selected regions for each band appear as red boxes on the image. (e) The MinPeakProm and Divisor values are specified, prompting the display of a corresponding red overlay. (f) The final results for the integrated bands are collected for data analysis.

materials and hazards, experimental methods, and discussion questions.

\section{Quantitative Image Analysis}

Quantitative image analysis of TLC plates may be conducted following the detailed tutorial presented in the Supporting Information. In short, once the elution and subsequent drying was completed, imaging was carried out with a mobile-phone camera. For the quantification of the fluorescent compound rhodamine $6 \mathrm{G}$, the plate was placed in a black box with a UV lamp at $254 \mathrm{~nm}$. These units contain a UV filter in the observation window to allow the investigation of the UVexcited fluorescent signal while reducing exposure to the UV light. In this example, the image was taken with a mobile phone through the eyepiece of the black box as shown in Figure 1c.

The image was subsequently uploaded to the quantitative TLC (qTLC) desktop software. A demonstration of the qTLC-software analysis can be found in Figure 2. For absorbance-based (i.e., nonfluorescent) quantification, the image colors were manually inverted such that the bands appeared bright on a dark background. This allowed the program to quantify nonfluorescent compounds in the same manner as fluorescent compounds. The user then cropped the image around the region containing the bands and specified the numbers of lanes and bands per lane for the given sample. Subsequently, the user coarsely identified the lanes by drawing rectangles and then subdivided these regions into the individual bands. The qTLC program then determined the signal over the background on the basis of the minimum peakprominence value provided ("MinPeakProm" in the desktop software and "Min Peak Prominence" in the webapp). The upper and lower boundaries of the band were established by the "Divisor" value. The recognized area was subsequently shown as a red overlay on the image of the TLC plate. The procedure was repeated for each band and the results were obtained by using the "Calculate" option in the qTLC software with the same MinPeakProm and Divisor values. Note that in the webapp, both the recognized area and the result are shown simultaneously. The data obtained for the reference dilutions then serves to construct a calibration curve for the quantification of the unknown sample.

We refer to the SI for a sample student handout on quantitative image analysis that contains learning objectives; theory; details on the analysis procedure, including image acquisition, use of the qTLC software, and signal integration with MinPeakProm and Divisor; and suitable discussion questions.

\section{HAZARDS}

Sudan IV can cause eye, skin, and respiratory irritation. Rhodamine 6G is very hazardous if ingested; it can cause eye, skin, and respiratory irritation and is hazardous if inhaled. Both acetic acid and methanol $(\mathrm{MeOH})$ are flammable liquids and vapors. Acetic acid is skin-corrosive. $\mathrm{MeOH}$ is toxic if ingested, inhaled, or absorbed through skin contact and can cause damage to organs. Silica from the TLC plates may cause mild eye and skin irritation and may cause irritation to the respiratory system. Users should consult the respective Material Safety Data Sheets of all chemicals, which are available from the supplier. Furthermore, the experimenters must wear eye protection, gloves, and lab coats and work in a fume hood. Care should be taken to minimize the production of and contact with the silica dust produced while handling the TLC plates.

\section{RESULTS AND DISCUSSIONS}

\section{qTLC Analysis and Results}

Once the band intensities are quantified, they are used to determine the concentration of the unknown sample in the 

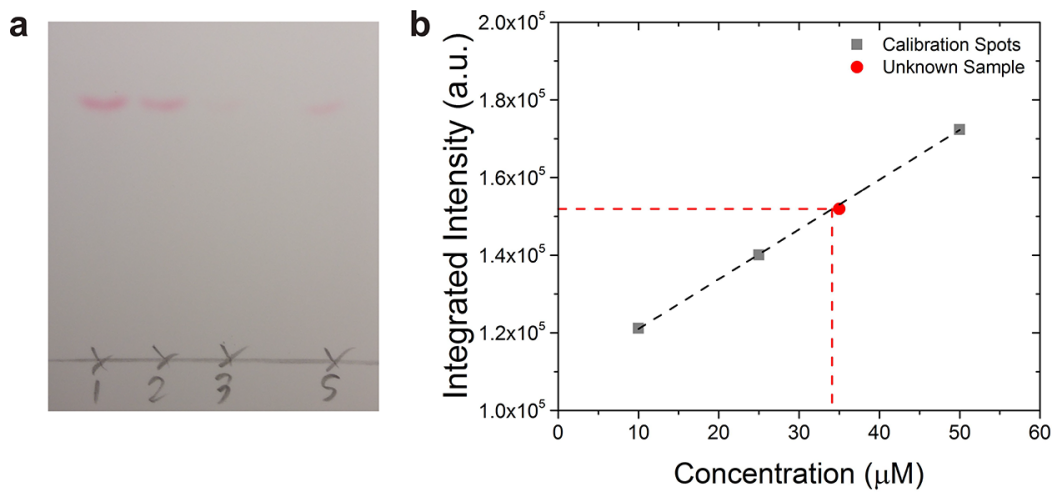

Figure 3. Absorbance-based quantification of Sudan IV. (a) Example digital photograph of the TLC plate after spotting, elution, and drying. (b) Plot of the integrated band intensities with the standard curve based on three calibration points. The obtained linear trend $\left(R^{2}=0.99998\right)$ allowed the determination of the concentration in an unknown sample (in this example $34.1 \mu \mathrm{M}$ ) based on the integrated intensity (actual concentration of $35.0 \mu \mathrm{M}, 2.6 \%$ error).
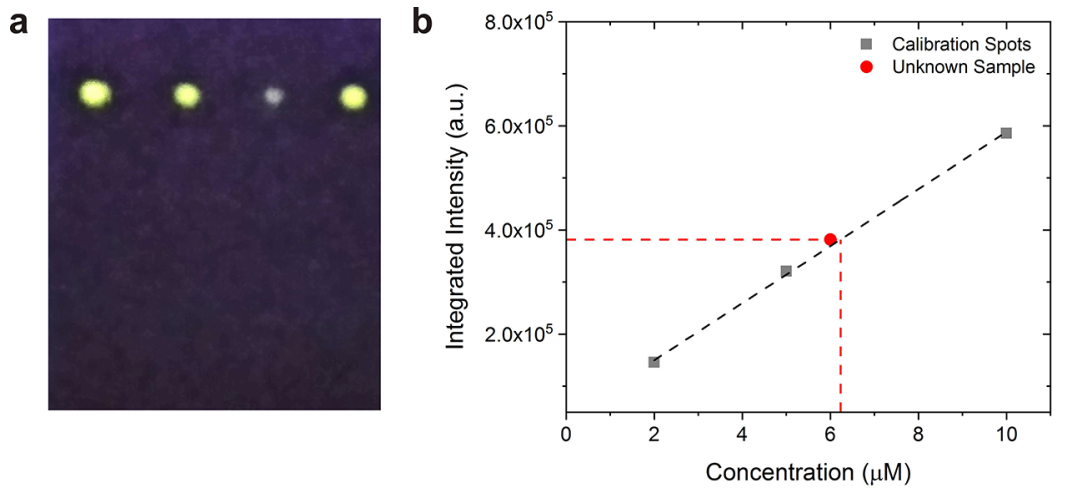

Figure 4. Fluorescence-based quantification of rhodamine 6G. (a) Example digital photograph of the TLC plate with three known concentrations of rhodamine $6 \mathrm{G}$ and the unknown sample. Imaging was carried out in a black box with an excitation wavelength of $254 \mathrm{~nm}$. (b) Plot of the integrated band intensities with the standard curve based on three calibration spots $\left(R^{2}=0.99967\right)$ and interpolation of the concentration of the unknown sample (in this example $6.2 \mu \mathrm{M}$ ) based on the integrated intensity (actual concentration of $6.0 \mu \mathrm{M}, 3.8 \%$ error).

example experiments. The integrated intensity of each band is therefore plotted against the known concentration of each sample. A linear trend is fitted to obtain a standard curve, and this is used to determine the concentration of the unknown sample on the basis of its integrated intensity. Including the calibration curve on the same plate as the unknown sample is an important step in this process in order to mitigate possible systematic experimental errors as well as plate-to-plate variability. Variations between TLC plates, stock solutions, mobile phases, or elution handling could otherwise detrimentally affect the quantification. We note that the calibration spots need to obey a linear trend, and the intensity of the unknown sample needs to lie within the linear regime. Otherwise, further dilutions are required for reliable interpolation (see the SI, Figures S7 and S8).

We demonstrate absorbance-based quantification for Sudan IV. This compound is an azo-dye widely used for staining biological samples and as a colorant in plastics, oils, emulsions, other hydrocarbon products, and industrial solvents. In Figure 3a, a sample digital photograph of the TLC plate is shown after spotting three known concentrations of the compound (spots 1-3) alongside the unknown sample (spot $S$ ), elution, and subsequent drying. The qTLC software was then used to quantitatively analyze the bands. The obtained intensity plotted against the respective concentration is shown in Figure 3b. A linear fit was applied to the obtained calibration data to produce a standard curve. The concentration of the unknown sample was subsequently determined by interpolation based on the obtained integrated peak intensity. In this example, an experimentally derived concentration of $34.1 \mu \mathrm{M}$ was found for the unknown sample, which was comparable to the actual concentration of $35.0 \mu \mathrm{M}(2.6 \%$ error $)$. Across nine runs, the average absolute error was found to be $6.5 \pm 3.7 \%$.

Rhodamine $6 \mathrm{G}$ is a common organic dye and serves here as an example for fluorescence-based quantification. The compound is used in many applications ranging from the labeling of biological materials to dye lasers, solar cells, paper products, and textiles. ${ }^{16,17}$ In Figure $4 \mathrm{a}$, an example digital photograph of the developed TLC plate is shown at an excitation wavelength of $254 \mathrm{~nm}$ with three known concentrations of the compound alongside the unknown sample. In Figure 4b, results are plotted for the integration of the bands using the qTLC software. On the basis of band integration and a linear calibration curve, the concentration of the unknown sample was determined to be $6.2 \mu \mathrm{M}$, which was comparable to the expected value of $6.0 \mu \mathrm{M}$ ( $3.8 \%$ error). Over nine runs, the average absolute error was found to be 7.0 $\pm 3.6 \%$.

\section{Digital Images}

The quality of the image can affect the reliability of the final results obtained by the qTLC software. The software integrates the bands on the basis of the intensity of the signal, so color is 
neglected. An RGB image is automatically converted to grayscale on the basis of a weighted sum of the RGB components. ${ }^{18}$ Effectively, the image is reduced to a $2 \mathrm{D}$ matrix, in which the intensity of light recorded by each pixel is represented by a value in the matrix. As the bands are identified by the user, the matrix is subdivided. During the integration process, the software moves across the submatrix, analyzing a column of data one pixel wide at a time. As such, a higher pixel density can improve the analysis. Another consideration is the bit depth of the image. A grayscale image with a bit depth of 8 can have intensity values in the range of 0 to 255, whereas a bit depth of 16 may exhibit values in the range of 0 to 65,535 . An image with a higher bit depth will thus have a higher degree of accuracy in the analysis. Our quantification routine by the qTLC software accounts for the two-dimensional area in which the spot is located in addition to the pixel intensity. Therefore, an image taken by a standard smart phone is typically more than sufficient for reliable quantification.

\section{Error Management}

There are a number of ways errors can be introduced into these experiments. As the determination of the concentration of the unknown sample is dependent on the volume and concentrations of the sample curve, any volumetric error in making these solutions or spotting them onto the TLC plate will affect the final results. Students should ensure they plan their dilutions carefully and that the volume handling is carried out as accurately as possible. If the spotting is accomplished with a pipette, the students need to take care that the same pipetting protocol is used for each spot (e.g., wetting the pipette first before every spot). If capillary tubes are employed for the spotting, the calculated weight of the actual sample volume can then be used to correct the integrated intensity of each spot.

The image analysis of the TLC plate is another potential source of error. Although the qTLC software is able to perform a background correction, results will be best if the plate is illuminated as evenly as possible. Any stray shadows from the camera or other apparatus may affect the results. Furthermore, extraneous material on the TLC plate may negatively affect the results. Students should take care to avoid stray marks or material (such as dust, silica particulates, or droplets from the sample) on the TLC plate. Lastly, the quantification process looks at the relative integrated intensities to determine the concentration of an unknown sample. As such, an image in which the bands are saturated will have less accuracy.

Please see the SI for a more comprehensive discussion on potential sources of error, including image-acquisition guidelines, information on how saturation can impact the results, signal linearity versus sample concentration, and image analysis using MinPeakProm and Divisor.

\section{Separation of Mixtures}

For more advanced lesson plans, mixtures of different compounds or more complex samples may be used. The same quantification process shown here can be applied by analyzing bands from individual components, allowing the students to quantify each component separately from one another and even identifying which compounds are present in an unknown sample. We note that separate calibration curves may be obtained for several target compounds by coaddition in the dilution experiments. Adequate elution and band separation must be verified in this case, and the mobilephase composition needs to be adjusted where required.

\section{CONCLUSIONS}

In conclusion, we present an intuitive and user-friendly analytic platform that allows students to reliably quantify the compounds they are studying by TLC using a standard mobile phone and a simple computer interface. The two experiments reported herein demonstrate that our free and purpose-built standalone software and equivalent webapp qTLC ${ }^{15}$ is capable of providing reliable quantitative data for both absorbancebased and fluorescence-based TLC read-outs. The experimental requirements are easily implemented in an educational laboratory setting and will allow students to not only observe the separation of the compounds being studied but also carry out quantitative analysis.

\section{ASSOCIATED CONTENT}

\section{S Supporting Information}

The Supporting Information is available on the ACS Publications website at DOI: 10.1021/acs.jchemed.8b00144.

Supporting Information with qTLC software and webapp tutorials; details on MinPeakProm, Divisor, the signal-integration process, and background subtraction; image-acquisition guidelines; choice of concentration range; and overview of experimental errors (PDF)

Student handout with instructions on experimental TLC (PDF)

Student handout with instructions on image analysis and data acquisition with the qTLC software (PDF)

Student handout with instructions on image analysis and data acquisition with the qTLC webapp (PDF)

All MATLAB components for customization and further development of the qTLC software (ZIP)

Links for installation of the qTLC software on MacOS with separate MATLAB Runtime installation, standalone installation of the qTLC software on MacOS, installation of the qTLC software on Windows with separate MATLAB Runtime installation, and standalone installation of the qTLC software on Windows (PDF)

\section{AUTHOR INFORMATION}

\section{Corresponding Author}

*E-mail: s.guldin@ucl.ac.uk. ORCID $\odot$

Niamh Mac Fhionnlaoich: 0000-0001-8459-9994

Luis A. Serrano: 0000-0001-5292-5150

Alaric Taylor: 0000-0001-6494-8309

Runzhang Qi: 0000-0002-7805-6501

Stefan Guldin: 0000-0002-4413-5527

\section{Author Contributions}

${ }^{\dagger}$ N.M.F. and S.I. contributed equally to this work. Notes

The authors declare no competing financial interest. The qTLC software can also be obtained from the corresponding author at s.guldin@ucl.ac.uk. 


\section{ACKNOWLEDGMENTS}

This project received funding from the European Union's Horizon 2020 Research and Innovation Programme under grant agreement no. 633635 (DIACHEMO). N.M.F. is grateful for support by the EPSRC under a Doctoral Training Partnership (EP/M507970/1). The authors acknowledge valuable feedback from Jin Hao and Wei Lik (University College London).

\section{REFERENCES}

(1) Spangenberg, B.; Poole, C. F.; Weins, C. Quantitative Thin-Layer Chromatography; Springer: Heidelberg, 2011; p 388.

(2) Hansen, S.; Pedersen-Bjergaard, S.; Rasmussen, K. Introduction to Pharmaceutical Chemical Analysis; John Wiley \& Sons, Ltd.: Chichester, U.K., 2011; pp 163-172.

(3) Dickson, H.; Kittredge, K. W.; Sarquis, A. M. Thin-Layer Chromatography: The "Eyes" of the Organic Chemist. J. Chem. Educ. 2004, 81, 1023-1025.

(4) Brinkman, U. A. T.; De Vries, G. Small-Scale Thin-Layer Chromatography. Experiments for Teaching Purposes. J. Chem. Educ. 1972, 49, 545 .

(5) Olesen, B.; Hopson, D. Identification of Unknown Black Inks by Thin-Layer Chromatography. J. Chem. Educ. 1983, 60, 232.

(6) Ma, Y.; Yeung, E. S. Determination of Components in Beverages by Thin-Layer Chromatography: An Undergraduate Analytical Chemistry Experiment. J. Chem. Educ. 1990, 67, 428.

(7) Torres y Torres, J. L.; Hiley, S. L.; Lorimor, S. P.; Rhoad, J. S.; Caldwell, B. D.; Zweerink, G. L.; Ducey, M. Separation of Caffeine from Beverages and Analysis Using Thin-Layer Chromatography and Gas Chromatography-Mass Spectrometry. J. Chem. Educ. 2015, 92, 900-902.

(8) Sjursnes, B. J.; Kvittingen, L.; Schmid, R. Normal and ReversedPhase Thin Layer Chromatography of Green Leaf Extracts. J. Chem. Educ. 2015, 92, 193-196.

(9) Valverde, J.; This, H.; Vignolle, M. Quantitative Determination of Photosynthetic Pigments in Green Beans Using Thin-Layer Chromatography and a Flatbed Scanner as Densitometer. J. Chem. Educ. 2007, 84, 1505

(10) Kerr, E.; West, C.; Kradtap Hartwell, S. Quantitative TLCImage Analysis of Urinary Creatinine Using Iodine Staining and RGB Values. J. Chromatogr. Sci. 2016, 54, 639-46.

(11) Hess, A. V. I. Digitally Enhanced Thin-Layer Chromatography: An Inexpensive, New Technique for Qualitative and Quantitative Analysis. J. Chem. Educ. 2007, 84, 842-847.

(12) Fichou, D.; Ristivojević, P.; Morlock, G. E. Proof-of-Principle of rTLC, an Open- Source Software Developed for Image Evaluation and Multivariate Analysis of Planar Chromatograms. Anal. Chem. 2016, 88, 12494-12501.

(13) Manthorpe, D. P.; Lockley, W. J. S. Digitally Enhanced Thin Layer Chromatography: Further Development and some Applications in Isotopic Chemistry. J. Labelled Compd. Radiopharm. 2013, 56, 544-552.

(14) Rasband, W. Image); U.S. National Institutes of Health, Bethesda, MD, 2018. Available at https://imagej.nih.gov/ij/.

(15) Qi, R.; Mac Fhionnlaoich, N.; Ibsen, S.; Taylor, A.; Guldin, S. qTLC: A Webapp to Quantify Chemical Compounds via Thin Layer Chromatography; University College London, 2018. Available at https://www.qtlc.app/.

(16) Hair, S. An introductory dye laser experiment. J. Chem. Educ. 1996, 73, A7-A9.

(17) Sabnis, R. W. Handbook of Fluorescent Dyes and Probes; John Wiley \& Sons, Inc.: Hoboken, NJ, 2015; pp 373-379.

(18) MATLAB rgb2gray; MathWorks, 2018. Available at https://uk. mathworks.com/help/matlab/ref/rgb2gray.html. 